\title{
MULTIVALENTLY CLOSE-TO-CONVEX FUNCTIONS
}

TOSHIO UMEZAWA

1. Introduction. The class of close-to-convex functions in $|z|<1$ introduced by Kaplan [1] is characterized by the property that there exists a function $\phi(z)$ analytic, schlicht and convex in $|z|<1$ for which $R\left[f^{\prime}(z) / \phi^{\prime}(z)\right]>0,|z|<1$. Such functions are necessarily schlicht.

Let

$$
f(z)=z+a_{2} z^{2}+\cdots+a_{n} z^{n}+\cdots
$$

be a function of this class. Then it has been pointed out by Reade [2] that the inequalities

$$
\left|a_{n}\right| \leqq n, \quad n=2,3, \cdots
$$

hold. This result extends (1.2) to a wider class than was previously considered [3].

In this paper we extend these results to the case of functions $p$ valent in the unit circle.

2. Preliminaries. Hereafter we consider mainly functions of the form

$$
f(z)=z^{q}+\sum_{n=q+1}^{\infty} a_{n} z^{n}, \quad 1 \leqq q \leqq p .
$$

Definition 1. The function $\phi(z)$ of the form (2.1) is said to be an element of the class $C(p), p$ a positive integer, if it is regular in $|z|<1$ and if there is a $\rho<1$ such that for all $r$ in the interval $\rho<r<1$

$$
G(r, \theta)=1+R\left(r e^{i \theta} \frac{\phi^{\prime \prime}\left(r e^{i \theta}\right)}{\phi^{\prime}\left(r e^{i \theta}\right)}\right)>0, \quad 0 \leqq \theta \leqq 2 \pi,
$$

and

$$
\int_{0}^{2 \pi} G(r, \theta) d \theta=2 \pi p
$$

This definition is due to Goodman [4]. It is shown in [4] that if $\phi(z) \in C(p)$, it maps $|z| \leqq r<1$ onto a locally convex region and that $\phi(z)$ is at most $p$-valent in $|z|<1$. 1957.

Received by the editors December 5, 1956 and, in revised form, January 31, 
Remark. Since

$$
1+R \frac{z \phi^{\prime \prime}(z)}{\phi^{\prime}(z)}=\frac{d \arg d \phi(z)}{d \arg d z}
$$

(2.2) is equivalent to

$$
d \arg d \phi(z)>0,
$$$$
|z|=r<1
$$

and (2.3) means that $\phi(z)$ has $p-1$ critical points in $|z|<1$.

Definition 2. The function $f(z)$ is said to be an element of the class $K(p), p$ a positive integer, if it is regular in $|z|<1$ and if there exists a function $\phi(z)$ of the class $C(p)$ for which $R\left[f^{\prime}(z) / \phi^{\prime}(z)\right]>0,|z|<1$ holds.

Such functions will be called close-to-convex $p$-valent functions. We shall prove that such functions are at most $p$-valent in $|z|<1$. For the purpose we need the following lemma due to the author [5].

LEMma 1. Let $f(z)$ be regular for a simply connected closed domain $D$ whose boundary $\Gamma$ consists of a regular curve and $f^{\prime}(z) \neq 0$ on $\Gamma$. Suppose that

$$
\int_{\Gamma} d \arg d f(z)=2 p \pi
$$

If we have for an arbitrary arc $C$ on the boundary $\Gamma$

$$
\int_{C} d \arg d f(z)<(2 p+1) \pi
$$

or

$$
\int_{C} d \arg d f(z)>-\pi,
$$

then $f(z)$ is at most p-valent in $D$.

To handle the coefficient problem and the deformation problem for the class $K(p)$ we need the following lemma due to [4] and [6].

Lemma 2. Let $\phi(z) \in C(p)$ of the form (2.1) have $p-q$ critical points $\beta_{1}, \beta_{2}, \cdots, \beta_{p-q} \neq 0$ in $|z|<1$. Then

$$
\phi^{\prime}(z) \ll q F(z) / z \quad \text { for }|z|<1
$$

and

$$
\left|\phi^{\prime}\left(r e^{i \theta}\right)\right| \leqq q F(r) / r \quad \text { for } r<1
$$


where $F(z)$ is defined by

$$
F(z)=\frac{z^{q}}{(1-z)^{2 p}} \prod_{j=1}^{p-q}\left(1+\frac{z}{\left|\beta_{j}\right|}\right)\left(1+z\left|\beta_{j}\right|\right) .
$$

\section{The main theorems.}

Theorem 1. If $f(z) \in K(p)$, then $f(z)$ is at most p-valent in $|z|<1$.

Proof. By Definition 2 there exists a function $\phi(z)$ of the class $C(p)$ such that, for $|z|<1$,

$$
R\left[f^{\prime}(z) / \phi^{\prime}(z)\right]=R[d f(z) / d \phi(z)]>0 .
$$

Since $\phi^{\prime}(z)$ has exactly $p-1$ roots in $|z|<1$ by Definition $1, f^{\prime}(z)$ must have the same roots as $\phi^{\prime}(z)$ by (3.1). Hence we have

$$
\int_{|z|=r} d \arg d f(z)=2 p \pi \quad \text { for } \rho<r<1 .
$$

Since $d f / d \phi \neq 0$ in $|z|<1$ by (3.1), arg $(d f / d \phi)$ is one-valued in $|z|<1$. On the other hand we have

$$
\arg d f=\arg \frac{d f}{d \phi}+\arg d \phi .
$$

Hence $\arg d f$ is also one-valued if we take a suitable branch of $\arg d \phi(z)$.

By taking these branches and by the assumption (3.1), we have

$$
-\pi / 2<\arg d f\left(z_{i}\right)-\arg d \phi\left(z_{i}\right)<\pi / 2
$$

and

$$
-\pi / 2<-\arg d f\left(z_{j}\right)+\arg d\left(\phi z_{j}\right)<\pi / 2
$$

for arbitrary points $z_{i}, z_{j}, i>j$ on $|z|=r$, sufficiently near to 1 . Hence we have

$$
-\pi<\arg d f\left(z_{i}\right)-\arg d f\left(z_{j}\right)-\left(\arg d \phi\left(z_{i}\right)-\arg d \phi\left(z_{j}\right)\right)<\pi
$$

where $2 p \pi \geqq \arg d \phi\left(z_{i}\right)-\arg d \phi\left(z_{j}\right)>0$ since $\phi(z) \in C(p)$. Thus we have

$$
-\pi<\arg d f\left(z_{i}\right)-\arg d f\left(z_{j}\right)<(2 p+1) \pi,
$$

which is equivalent to

$$
-\pi<\int_{z_{j}}^{z_{i}} d \arg d f(z)<(2 p+1) \pi .
$$


(3.2) and (3.3) show that $f(z)$ is at most $p$-valent in $|z|<1$ by Lemma 1.

Theorem 2. Let

$$
f(z)=z^{q}+\sum_{n=q+1}^{\infty} a_{n} z^{n}
$$

be a function of the class $K(p)$. Suppose that in addition to the $(q-1)$ th order critical points at $z=0$ the function $f(z)$ has exactly $p-q$ critical points $\beta_{1}, \beta_{2}, \cdots, \beta_{p-q}$ such that $0<\left|\beta_{j}\right|<1, j=1,2, \cdots, p-q$. Then

$$
\begin{array}{rlr}
\left|a_{n}\right| & \leqq C_{n}, & n=q+1, q+2, \cdots, \\
\left|f\left(r e^{i \theta}\right)\right| & \leqq F(r) & \text { for } r<1
\end{array}
$$

and

$$
\left|f^{\prime}\left(r e^{i \theta}\right)\right| \leqq F^{\prime}(r)
$$$$
\text { for } r<1
$$

where $C_{n}$ and $F(r)$ are defined by

$$
\begin{aligned}
F(z) & =q \int_{0}^{z} \frac{t^{q-1}(1+t)}{(1-t)^{2 p+1}} \sum_{j=1}^{p-1}\left(1+\frac{t}{\left|\beta_{j}\right|}\right)\left(1+t\left|\beta_{j}\right|\right) d t \\
& =z^{q}+\sum_{n=q+1}^{\infty} c_{n} z^{n}
\end{aligned}
$$

Proof. By our hypothesis, there exists a function of the class $C(p)$ which has the same form and the same critical points as $f(z)$ such that $R\left[f^{\prime}(z) / \phi^{\prime}(z)\right]>0$ for $|z|<1$. Hence by Carathéodory's theorem

$$
\frac{f^{\prime}(z)}{\phi^{\prime}(z)} \ll \frac{1+z}{1-z}
$$

and by Strohhäcker's theorem [7]

$$
\frac{1-r}{1+r} \leqq\left|\frac{f^{\prime}\left(r e^{i \theta}\right)}{\phi^{\prime}\left(r e^{i \theta}\right)}\right| \leqq \frac{1+r}{1-r} \quad \text { for } r<1 .
$$

On the other hand we have (2.8) and (2.9) for $\phi^{\prime}(z)$. Accordingly we have (3.7) and

$$
f^{\prime}(z) \ll \frac{z^{q-1}(1+z)}{(1-z)^{2 p+1}} \prod_{j=1}^{p-q}\left(1+\frac{z}{\left|\beta_{j}\right|}\right)\left(1+z\left|\beta_{j}\right|\right) .
$$

Hence we have (3.5) and (3.6) with (3.8). q.e.d. 
CoROllary 1. If $f(z)=z^{p}+\sum_{n=p+1}^{\infty} a_{n} z^{n} \in K(p)$, then $f(z)$ is $p$ valent for $|z|<1$ and the inequalities

$$
\begin{aligned}
& \left|a_{n}\right| \leqq\left(\begin{array}{c}
n+p-1 \\
2 p-1
\end{array}\right), \quad n=p+1, p+2, \cdots, \\
& \frac{r^{p}}{(1+r)^{2 p}} \leqq\left|f\left(r e^{i \theta}\right)\right| \leqq \frac{r^{p}}{(1-r)^{2 p}} \quad \text { for } r<1 \\
& \left|a_{n}\right| \leqq\left(\begin{array}{c}
n+p-1 \\
2 p-1
\end{array}\right), \quad n=p+1, p+2, \cdots,
\end{aligned}
$$

and

$$
\frac{p r^{p-1}(1-r)}{(1+r)^{2 p+1}} \leqq\left|f^{\prime}\left(r e^{i \theta}\right)\right| \leqq \frac{p r^{p-1}(1+r)}{(1-r)^{2 p+1}} \quad \text { for } r<1
$$

hold.

Proof. (3.10) and the upper bounds in (3.11) and (3.12) are obvious. Let us prove the lower bounds in them.

In this special case, for the corresponding convex $p$-valent function $\phi(z)$, we have

$$
\frac{p r^{p-1}}{(1+r)^{2 p}} \leqq\left|\phi^{\prime}\left(r e^{i \theta}\right)\right|
$$

since $z \phi^{\prime}(z)$ is $p$-valently starlike in $|z|<1$, [4]. Hence we have the lower bound for $\left|f^{\prime}(z)\right|$ in (3.12) from (3.9).

Let $w$ be one of the nearest points from the origin to the image curve of $|z|=r<1$ under $f(z)$. Further let $L$ be the curve on the $z$-plane corresponding to the line segment $0 w$. Then we have

$$
\begin{aligned}
|f(z)| & =\int_{L}\left|f^{\prime}(z)\right||d z| \geqq \int_{0}^{|z|}\left|f^{\prime}(z)\right| d|z| \\
& \geqq \int_{0}^{|z|} \frac{p|z|^{p-1}(1-|z|)}{(1+|z|)^{2 p-1}} d|z|=\frac{|z|^{p}}{(1+|z|)^{2 p}},
\end{aligned}
$$

which gives the lower bound in (3.10).

But we remark that the inequalities (3.11) follow at once from the $p$-valency of $f(z)$ by the result due to W. K. Hayman [8].

CoROllaRY 2. Let $f(z)=z^{p}+\sum_{n=p+1}^{\infty} a_{n} z^{n}$ be regular for $|z|<1$. Suppose that $f(z)$ satisfies one of the following conditions:

$$
\begin{array}{ll}
R\left[z f^{\prime}(z) / f(z)\right]>0 & \text { for }|z|<1 . \\
R\left[f^{\prime}(z) / z^{p-1}\right]>0 & \text { for }|z|<1 . \\
R\left[(1-z)^{p} f^{\prime}(z) / z^{p-1}\right]>0 & \text { for }|z|<1 . \\
R\left[\left(1-z^{p}\right) f^{\prime}(z) / z^{p-1}\right]>0 & \text { for }|z|<1 .
\end{array}
$$




$$
\begin{array}{ll}
R\left[(1-z)^{2 p} f^{\prime}(z) / z^{p-1}\right]>0 & \text { for }|z|<1 . \\
R\left[\left(1-z^{p}\right)^{2} f^{\prime}(z) / z^{p-1}\right]>0 & \text { for }|z|<1 . \\
R\left[\left(1-z^{2}\right)^{p} f^{\prime}(z) / z^{p-1}\right]>0 & \text { for }|z|<1 . \\
R\left[\left(1-z^{2 p}\right) f^{\prime}(z) / z^{p-1}\right]>0 & \text { for }|z|<1 .
\end{array}
$$

Then $f(z)$ is p-valent for $|z|<1$, and (3.10), (3.11) and (3.12) hold.

Proof. Under the condition (1), $\phi(z)=p \int_{0}^{z}(f(z) / z) d z \in C(p)$ and $R\left[f^{\prime}(z) / \phi^{\prime}(z)\right]=R\left[z f^{\prime}(z) / f(z)\right] / p>0$ for $|z|<1$. Hence $f(z) \in K(p)$.

In the other cases, $f(z) \in K(p)$, since the following functions
(2) $z^{p}$
(3) $\int_{0}^{z} \frac{z^{p-1}}{(1-z)^{p}} d z$
(4) $\int_{0}^{z} \frac{z^{p-1}}{1-z^{p}} d z$
(5) $\int_{0}^{z} \frac{z^{p-1}}{(1-z)^{2 p}} d z$
(6) $\int_{0}^{z} \frac{z^{p-1}}{\left(1-z^{p}\right)^{2}} d z$
(7) $\int_{0}^{z} \frac{z^{p-1}}{\left(1-z^{2}\right)^{p}} d z$,
(8) $\int_{0}^{z} \frac{z^{p-1}}{1-z^{2 p}} d z$

are easily seen to be $p$-valently convex in $|z|<1$.

Hence our conclusion follows in every case by Corollary 1 .

Here we note that in almost every case in Corollary 2 the inequalities can be sharpened considerably by direct use of Carathéodory's and Strohhäcker's Theorems.

\section{REFERENCES}

1. W. Kaplan, Close-to-convex schlicht functions, Michigan Mathematical Journal vol. 1 (1952) pp. 169-185.

2. M. Reade, Sur une classe de fonctions univalentes, C. R. Acad. Sci. Paris vol. 239 (1954) pp. 1758-1759.

3. M. S. Robertson, Analytic functions starlike in one direction, Amer. J. Math. vol. 58 (1936) pp. 465-472.

4. A. W. Goodman, On the Schwarz-Christoffel transformation and p-valent functions, Trans. Amer. Math. Soc. vol. 68 (1950) pp. 204-223.

5. T. Umezawa, On the theory of univalent functions, Tôhoku Math. J. vol. 7 (1955) pp. 212-223.

6. - A class of multivalent functions with assigned zeros, Proc. Amer. Math. Soc. vol. 3 (1952) pp. 813-820.

7. E. Strohhäcker, Beiträge zur Theorie der schlichten Funktionen, Math. Zeit. vol. 37 (1933) pp. 356-380.

8. W. K. Hayman, Symmetrization in the theory of functions, Technical Report No. 11, Contract N6-ORI-106, Task Order 5(NR-043-992), Office of Naval Research, Washington, D. C.

Gumma University, Maebashi, Japan 